\title{
ON A CONJECTURE OF A. J. HOFFMAN
}

\section{JOSEPH ZAKS}

ABSTRACT. A 3-polytope $P$ and four closed convex sets $C_{1}, \cdots$, $C_{4}$ in $P$ are described, having the following property: every line which meets $P$ meets at least one of the $C_{i}$ 's, and for every collection of polytopes $D_{1}, \cdots, D_{4}$, with $D_{i} \subseteq C_{i}$ for all $1 \leqq i \leqq 4$, there exists a line which meets $P$ and misses all of the $D_{i}$ 's. This is a counterexample to a conjecture of A. J. Hoffman.

The following question was recently raised by A. J. Hoffman [2]: "If $P$ is a $d$-polytope, $t>0$ an integer and $C_{1}, \cdots, C_{k}$ closed convex sets in $P$, such that every $t$-flat that meets $P$ meets $\cup_{i=1}^{k} C_{i}$; do there exist polytopes $D_{1}, \cdots, D_{k}$, with $D_{i} \subseteq C_{i}$ for all $1 \leqq i \leqq k$, such that if a $t$-flat meets $P$, it meets $\cup_{i=1}^{k} D_{i}$ ?"

The purpose of this note is to give a counterexample to this conjecture, with $d=3, t=1$ and $k=4$ (see Remarks 1 and 2).

A $d$-polytope here means the convex hull of a set of finitely many points in the Euclidean $d$-space $E^{d}$, having a nonempty interior, see [1].

Let $P$ be the polytope on the following six vertices in $E^{3}: A_{1}$ $=(0,0,0), A_{2}=(3,0,0), A_{3}=(0,3,0), A_{4}=(0,0,20), A_{5}=(3,0,20)$ and $A_{6}=(0,3,20)$.

$P$ is a prism with a base $B_{1}$ at the level $z=0$ and a base $B_{2}$ at the level $z=20$, where

$$
B_{1}=\text { convex hull }\left\{A_{1}, A_{2}, A_{3}\right\} \text { and } B_{2}=\text { convex hull }\left\{A_{4}, A_{5}, A_{6}\right\} \text {. }
$$

Let $L_{1}\left(L_{2}\right)$ be the line passing through the points $(0,0,1)$ and $(1,0,1),\left((0,0,3)\right.$ and $(0,1,3)$, resp.). Let $T_{1}\left(T_{2}\right)$ be the cylinder containing all points within a distance of $\leqq 1$ to $L_{1}\left(L_{2}\right.$, resp.).

We define $C_{i}, 1 \leqq i \leqq 3$, as follows

$$
\begin{aligned}
& C_{1}=\text { convex hull }\left[\left(T_{1} \cap P\right) \cup\left\{A_{1}, A_{2}, A_{4}, A_{5}\right\}\right], \\
& C_{2}=\text { convex hull }\left[\left(T_{2} \cap P\right) \cup\left\{A_{1}, A_{3}, A_{4}, A_{6}\right\}\right], \\
& C_{3}=\text { convex hull }\left\{A_{2}, A_{3}, A_{5}, A_{6}\right\} .
\end{aligned}
$$

$C_{4}$ is defined as a subset of $B_{1}$, consisting of all the points $M$ in $B_{1}$,

Presented to the Society, March 27, 1970; received by the editors January 2, 1970.

AMS 1969 subject classifications. Primary 5210, 5230; Secondary 5090.

Key words and phrases. Convex $d$-polytype, closed convex set, affine $t$-flat, convex planar curve.

Copyright (C) 1971. American Mathematical Society 
for which there exists a point $N$ in $B_{2}$, such that the segment $M N$ has distance $\geqq 1$ to both of the two lines $L_{1}$ and $L_{2}{ }^{1}$

$C_{1}, \cdots, C_{4}$ were so chosen as to assure that if a line meets $P$, it meets $\mathrm{U}_{i=1}^{4} C_{i}$.

To justify our claim, it will suffice to prove the following

Lemma 1. $C_{4}$ is a closed convex set which is not a polytope.

Lemma 2. If $D_{i}$ is a polytope in $C_{i}$, for all $1 \leqq i \leqq 4$, then there exists a line $E$, such that $E \cap P \neq \varnothing$ and $E \cap\left(\cup_{i=1}^{4} D_{i}\right)=\varnothing$.

Proof of Lemma 1. If $M=(x, y, 0)$ is a point in $C_{4}$, then for some point $N$ of $B_{2}$ the segment $M N$ does not meet $T_{1}$ and $T_{2}$, or else it is tangent to one, or both of them. We observe that if a point $N$ exists, then another such point can be chosen to be a point of the edge $A_{5} A_{6}$, hence of the form $(3 \lambda, 3(1-\lambda), 20)$ for some $0 \leqq \lambda \leqq 1$.

Let $\alpha$ be a subset of the positive orthant of the $x y$-plane, consisting of all the points $M=(x, y, 0)$, for which there exists a value of $\lambda$, $0 \leqq \lambda \leqq 1$, such that the segment $M N$, where $N=(3 \lambda, 3(1-\lambda), 20)$, is tangent to both $T_{1}$ and $T_{2}$.

Since $M N$ is tangent to $T_{1}$, it follows that

$$
9 y^{2}-3 \lambda y+3 y=10
$$

(by setting the distance of the line passing through $M N$ to the line $L_{1}$ be equal to 1 ).

Similarly, since $M N$ is tangent to $T_{2}$, it follows that

$$
36 x^{2}+39 x \lambda+9 \lambda^{2}=50 .
$$

Let $\left(x_{\lambda}, y_{\lambda}\right)$ be the positive solution of the equations (1) and (2), for every $0 \leqq \lambda \leqq 1$. It follows easily that $x_{\lambda} \geqq x_{1}$ and $y_{\lambda} \geqq y_{0}$ for all $0 \leqq \lambda \leqq 1$.

Moreover, the boundary of $C_{4}$ is the union of the following three segments: $\left\{\left(x, y_{0}\right) \mid x_{0} \leqq x \leqq 3-y_{0}\right\}, \quad\left\{\left(x_{1}, y\right) \mid y_{1} \leqq y \leqq 3-x_{1}\right\}$ and $\left\{(x, y) \mid x \geqq x_{1}, y \geqq y_{0}\right.$ and $\left.x+y=3\right\}$, together with the arc $\alpha$, represented parametrically and implicitly by (1) and (2), with $0 \leqq \lambda \leqq 1$.

To show that $C_{4}$ is convex, it is obviously enough to prove that $\alpha$ is a convex curve, i.e., that $d^{2} y / d x^{2}>0$ at every point of $\alpha$.

This is a straightforward computation, which we outline: (1) implies (3) and (4), while (2) implies (5) and (6), where:

$$
\frac{d x}{d \lambda}=-\frac{13 x+6 \lambda}{24 x+13 \lambda}
$$

\footnotetext{
${ }^{1}$ Suppose there is a candle at each point of $B_{2}$, and every ray passing through the interior of $T_{1}$ and $T_{2}$ is absorbed; $C_{4}$ is then the illuminated part of $B_{1}$.
} 


$$
\begin{aligned}
\frac{d^{2} x}{d \lambda^{2}} & =\frac{25 x-25 \lambda(d x / d \lambda)}{(24 x+13 \lambda)^{2}}, \\
\frac{d y}{d \lambda} & =\frac{y}{6 y-\lambda+1}, \\
\frac{d^{2} y}{d \lambda^{2}} & =\frac{y+(1-\lambda) d y / d \lambda}{(6 y-\lambda+1)^{2}} .
\end{aligned}
$$

Since $x>0, y>0$ and $0 \leqq \lambda \leqq 1$, it follows from (3) that $d x / d \lambda<0$, and from (5) that $d y / d \lambda>0$; these, in turn, imply that $d^{2} x / d \lambda^{2}>0$ and $d^{2} y / d \lambda^{2}>0$.

Using the well-known formula

$$
\frac{d^{2} y}{d x^{2}}=\left(\frac{d x}{d \lambda} \cdot \frac{d^{2} y}{d \lambda^{2}}-\frac{d y}{d \lambda} \cdot \frac{d^{2} x}{d \lambda^{2}}\right) /\left(\frac{d x}{d \lambda}\right)^{3}
$$

it follows immediately that $d^{2} y / d x^{2}>0$ on $\alpha$. Moreover, $d^{2} y / d x^{2}>0$ implies that $\alpha$ is not a segment.

As a result, $C_{4}$ is a closed convex set which is not a polytope, and the proof of Lemma 1 is completed.

Proof of Lemma 2. Let $D_{1}, \cdots, D_{4}$ be polytopes, with $D_{i} \subseteq C_{i}$ for all $1 \leqq i \leqq 4$.

$D_{4}$ is a convex (planar) polygon in $C_{4}$, and $C_{4}$ is, by Lemma 1 , a closed convex (planar) set which is not a polygon. Therefore, there exists a point $R$ on the arc $\alpha$, such that $R \notin D_{4}$. Since $D_{4}$ is convex, there exists the point $R^{*}$, nearest to $R$ in $D_{4}$. The point $S=\frac{1}{2}\left(R+R^{*}\right)$ is an interior point of $C_{4}$ and $S \notin D_{4}$.

Let $S^{\prime}$ be a point in the upper base $B_{2}$ of $P$, such that the segment $S S^{\prime}$ is of distance $\geqq 1$ from the two lines $L_{1}$ and $L_{2}$. Since $S$ belongs to the interior of $C_{4}$, we may assume, without loss of truth, that $S^{\prime}$ belongs to the interior of $B_{2}$.

Let $S_{1} S_{1}^{\prime}$ be a segment, obtained from $S S^{\prime}$ by an $\epsilon$-push towards the face $C_{3}$ of $P$, such that $S_{1} S_{1}^{\prime} \| S S^{\prime}, S_{1}$ is an interior point of $C_{4}-D_{4}, S_{1}^{\prime}$ is an interior point of $B_{2}$ and $S_{1} S_{1}^{\prime}$ is of distance $>1$ from both $L_{1}$ and $L_{2}$.

The line $E$, containing the segment $S_{1} S_{1}^{\prime}$, is such that $E \cap P \neq \varnothing$ and $E \cap\left(\cup_{i=1}^{4} D_{i}\right)=\varnothing$.

This completes the proof of Lemma 2.

REMARK 1. The original example, as presented to the Society, involved $k=10$ (with $d=3$ and $t=1$ ); a remark by (a student of) A. J. Hoffman reduced it to $k=6$; that remark enabled us to further reduce the value of $k$ to 4 . 
REMARK 2. By taking the Cartesian product of our example with the $q$-dimensional cube $I^{q}$, we derive additional counterexamples in the cases where $d=q+3$ and $t=q+1$, for all $q \geqq 1$.

\section{REFERENCES}

1. B. Grünbaum, Convex polytopes, Pure and Appl. Math., vol. 16, Interscience, New Ycrk, 1967. MR 37 \#2085.

2. A. J. Hoffman, On covering of polyhedra by polyhedra, Proc. Amer. Math. Soc. 23 (1969), 123-126. MR 40 \#835.

Wayne State University, Detroit, Michigan 48202 